\title{
Criterios de calidad de una buena práctica para la aplicación de las TIC a nivel de institución
}

\author{
Quality criteria of best practice for the \\ implementation of ICT - level institution
}

\author{
Ramón F. FERREIRO GRAVIE \\ Nova Southeastern University, EEUU
}

Recibido: Diciembre 2011

Aceptado: Abril 2012

\section{Resumen}

El artículo presenta una lista de criterios de calidad para una buena práctica en la aplicación de las TIC en instituciones latinoamericanas. La reflexión se hace a partir de los resultados de una consulta a expertos en los cursos escolares 2008-2009 y 2009-2010 entre profesores, investigadores y líderes de educación de América Latina y el Caribe. Las respuestas, comentarios y sugerencias permitieron la elaboración de un documento de trabajo como evidencia exploratoria al estudio experimental aún en proceso sobre nuevos ambientes de aprendizajes.

Palabras clave: Buenas Prácticas, Tecnologías de la Información y la Comunicación (TIC), Educación a Distancia, Nuevos Ambientes de Aprendizajes.

\begin{abstract}
The article presents a list of criteria of quality for best practice in the application of ICTs in Latin American institutions. Reflection is done based on the results of a inquiry to experts in school courses 2008-2009 and 2009-2010 among teachers, researchers and leaders of education of Latin America and the Caribbean. Responses, comments and suggestions allowed the preparation of a working paper as exploratory evidence still undergoing experimental study on new learning environments.
\end{abstract}

Keywords: Best Practices, ICT, Distance Education, New Learning Environments.

\section{Las buenas prácticas}

El concepto de "buena práctica" constituye un constructo muy frecuente en la literatura educativa de Norteamérica. Su empleo se ha realizado fundamentalmente en tres direcciones diferentes:

1. Una tiene que ver con la identificación de prácticas exitosas a partir de indicadores de logros de objetivos y rendimiento académicos. 
2. La otra consiste en poner en práctica y valorar experiencias exitosas de otros países, regiones o bien instituciones, registrados en la literatura científica, y a partir de ello determinar su eficiencia y eficacia.

3. Un tercer criterio de "buena práctica" es la de documentar y sistematizar la percepción que tienen los protagonistas de un movimiento de cambio sobre cómo introducir, desarrollar o bien valorar una innovación en el amplio sentido de la palabra.

Hace algunos años Chickering y Gamson (citados en Newberry, 2001) definieron siete principios de una enseñanza presencial eficaz, dado que permiten la creación de ambientes de aprendizaje que aseguran un alto nivel de interacción, cooperación y comunicación. Estos tres conceptos (interacciones, cooperación y comunicación) constituyen en nuestro criterio piedras angulares para el diseño educativo a nivel de aula, no obstante estar condicionado por toda una serie de factores que dependen en gran medida de tomas de decisiones a nivel de institución.

Años más tarde con el auge de las TIC y los movimientos de integración de éstas a los procesos formales de enseñanza, Chickering y Ehrmann (citados en Collins-Brown, 2001) plantearon siete principios de las buenas prácticas de aplicación de las TIC a la educación que se pueden resumir en que las TIC deben:

1. Favorecer la relación entre profesores y estudiantes. Las TIC amplían y diversifican las vías y modos de comunicación entre los agentes personales del proceso de enseñanza. Basta tener presente el papel que puede jugar por ejemplo el correo electrónico o bien los foros y charlas (chat) electrónicos, así como el empleo cada vez mayor del Sky.

2. Propiciar la cooperación entre los estudiantes. La tecnología permite a los estudiantes bajo la orientación del profesor participar en pequeños equipos de estudio y trabajo en línea y desarrollar proyectos en colaboración, sin coincidir necesariamente en espacio y tiempo.

3. Estimular el aprendizaje activo y reflexivo de búsqueda de información y construcción social del conocimiento. La Web y el uso por ejemplo de software de simulación entre otras aplicaciones tecnológicas permiten como nunca antes adquirir y comprender conceptos de gran nivel de complejidad por las abstracciones que implican. En el plano económico el empleo de software interactivos y de simuladores pueden significar ahorros considerables de dinero en cursos eminentemente prácticos y de laboratorios como en química, física y otras materias escolares.

4. Proporcionar retroalimentación oportuna. La tecnología permite dar respuestas casi inmediatas a los estudiantes, sobre preguntas, tareas y otras actividades del curso. Todo va a estar en función entre otros factores del diseño didáctico que se emplea y los objetivos que se persiguen. 
5. Contribuir a flexibilizar los períodos y plazos de entrega, lugares de trabajo, etc. El uso de la tecnología favorece la atención personalizada de los estudiantes y permite entre otras cosas por ejemplo trabajar en casa (o desde cualquier lugar) y en el horario que les sea conveniente.

6. Comunicar altas expectativas, por ejemplo, los software de simulación proporcionan diferentes tipos de problemas de aprendizaje o bien la visión desde distintos ángulos o perspectivas. Las TIC por lo regular hacen público el trabajo de los estudiantes (en un foro de discusión, mediante video "subidos" a YouTube) que entre otras cosas contribuye a elevar el nivel de expectativas de los estudiantes.

7. Respetar los diversos talentos y estilos y ritmos de aprendizaje. La tecnología ofrece múltiples sistemas de entrega, que proveen a los estudiantes de diferentes métodos que se adaptan mejor a su estilo de aprendizaje.

Otras muchas referencias hacen explícito el valor metodológico y práctico del concepto de "buenas prácticas" en cuanto al empleo de las TIC y el diseño de nuevos ambientes de aprendizajes, como constructo a partir del cual podemos trabajar para precisar lo deseable en estos entornos de crecimiento personal (Roger, 1995; Sorensen y Baylen, 2009; Orellana, 2009). Aunque como en todo proceso educativo estará relacionado con otros factores y variables que los condicionan y determinan a uno u otro nivel.

En tal sentido Hernández (2008), plantea que el aprendizaje empleando TIC es efectivo si cumple con cuatro condiciones que en síntesis son: 1-entorno creativo que incluya múltiples herramientas y materiales (sonido, imágenes, videos...) e implique al estudiante en la adquisición del conocimiento, logrando un compromiso activo de cada integrante del aula; 2- relaciones profesor - estudiante y de estos entre sí y con el contenido de enseñanza; 3 - realización de trabajo en equipo y 4 relación del estudiante con el mundo real.

En los países latinoamericanos desde los años noventa se han incrementado las experiencias pedagógicas en la aplicación de las TIC tanto en ambientes presenciales como a distancia, y en ambientes "combinados" o mixtos (blended). No obstante aún se discute sobre su nivel de pertinencia, los tipos de tecnologías a introducir, la preparación de los profesores y entre otros aspectos la calidad de los procesos y resultados obtenidos con la aplicación de las TIC tanto en la enseñanza presencial como a distancia.

Muchas de estas experiencias no han tenido el éxito esperado pese a la motivación, el dominio del contenido por parte del profesor y la probada calidad de las tecnologías empleadas, entre otros, por no estar determinadas a nivel de institución determinadas política y normativa al respecto que las respalden y estimulen (Ferreiro, 2011).

La aplicación de las TIC ha sido en nuestro Continente más una iniciativa de profesores aislados que de las instituciones. La ausencia de políticas y modelos educativos que consideren las TIC como elemento sustantivo del mismo es un factor a considerar cuando se valora el éxito y la trascendencia de las experiencias en nuestros países.

En tal sentido resulta conveniente la precisión de los criterios que poseen un conjunto de personas que en la práctica diaria están trabajando en pos de hacer posible el 
correcto empleo de las TIC a nivel de institución de modo tal que lo realizado a nivel de aula por los maestros no tan solo sea viable sino también resulten buenas prácticas por el logro de los objetivos educativos planteados.

\section{Propósito del artículo}

El propósito de este artículo es presentar una lista de criterios de calidad (indicadores) y determinadas reflexiones para la correcta introducción y aplicación de las TIC en las instituciones educativas a partir de datos obtenidos en un estudio de mayor alcance y en proceso de ejecución (Ferreiro, 2011) aún no publicado.

En el estudio antes mencionado entre otras actividades realizadas e instrumentos aplicados se realizó una encuesta internacional sobre el concepto de "buenas prácticas" en particular a la aplicación de las TIC para el diseño y puesta en práctica de nuevos ambientes de aprendizajes, en la tercera dirección mencionada arriba.

Partimos del criterio que la opinión de personas expertas, debe caracterizar como tendencia, una buena práctica a nivel institucional en cuanto a la introducción y al empleo de las tecnologías para aprender. En otras palabras el "deber ser" en un momento determinado de esta tendencia educativa que pone énfasis en las múltiples posibilidades de aplicación de las TIC a los procesos de aprendizajes y enseñanza desde la perspectiva del nivel central o institucional, en otras palabras lo que la escuela, la universidad o centro debe tomar en consideración para hacer posible la correcta integración de las TIC a los procesos educativos formales.

Conocer, sistematizar y documentar los criterios de personas expertas, sus experiencias y valoraciones, permite entre otros aspectos tener un punto de referencia para justificar, fundamentar, proyectar lo que estamos haciendo o queremos hacer y lograr en los distintos niveles de una institución educativa en pos de la formación del ciudadano del siglo XXI.

En la investigación que nos sirve de punto de partida un total 14 aspectos fueron los identificados como criterios de calidad de una buena práctica para la aplicación de las TIC a los procesos de enseñanza presenciales y de educación a distancia a nivel institucional de modo tal que los mismos hagan posible la aplicación e integración de las tecnologías a los mismos.

El resultado obtenido (Ferreiro, 2011) lo hemos empleado en talleres de capacitación y reflexión lo que nos ha permitido entre otras cosas esclarecer posiciones, justificar y fundamentar decisiones, y lo que es más importante perfeccionar procesos de asesoramiento y diseño de la práctica de la educación.

En el trabajo realizado se reconoció como experto en aplicación de las TIC a toda aquella persona que se desempeña en alguna institución latinoamericana donde se declara enseñar total o parcialmente empleando de alguna manera las TIC o mediante la modalidad de educación a distancia. Los expertos considerados mantienen en su comunidad un prestigio dado entre otras cosas por su participación en congresos, tener 
publicaciones, membrecía en asociaciones profesionales, y ejerce de un modo u otro un liderazgo en cuanto a la integración de las TIC al proceso de aprendizaje - enseñanza.

Para el desarrollo de esa tarea de investigación se confeccionó una lista de expertos, a partir de la definición adoptada, de personas reconocidas por su trabajo al respecto y cuyos correos electrónicos o número de teléfonos y fax teníamos o podíamos obtener. En total se enlistaron 95 personas expertas pertenecientes a distintos países, instituciones y asociaciones. Del total encuestado recibimos 52 respuestas para un $54.7 \%$ (Ferreiro, 2011).

En el estudio citado se emplearon distintos instrumentos tales como cuestionario, entrevista personales, grupos focales, ensayo y análisis de contenido en distintos momento del desarrollo del trabajo. La información recolectada se procesó mediante la técnica de "análisis de contenido" presentándose a continuación en orden de lo más general a lo particular (Ferreiro, 2011).

A las personas enlistadas se le envió un mensaje electrónico y posteriormente una carta identificándonos como Abraham S. Fischler School of Education de Nova Southeastern University y presentando a su consideración las tareas de investigación sobre generación net y nuevos ambientes de aprendizaje, y sobre esta sub tarea de investigación que el grupo de trabajo está realizando, dándoles a conocer el propósito de la consulta: Obtener su opinión sobre lo que para él/ella es requisito o condición necesaria previa para una buena práctica de empleo de las TIC en ambientes presenciales, educación a distancia o ambientes "combinados" a nivel institucional.

A los miembros de la muestra que respondieron el cuestionario se les invitó a seguir contribuyendo y a constituir una sub muestras por regiones y países (34 del total que respondieron) se les pidió una entrevista, o bien participar en una sesión de focus group o escribir un ensayo contándose con el apoyo de los 34 expertos en alguna de las modalidades de profundización empleadas.

\section{Criterios de calidad}

Los resultados sobre los requisitos y/o condiciones para una buena práctica en el empleo de las TIC en los procesos de enseñanza, y de educación a distancia, fueron por orden de generalidad los siguientes (Ferreiro, 2011):

1. Inscribir los esfuerzos de aplicación de las TIC en un plan o programa estratégico de transformación institucional que justifique la inversión que se realiza y precise las metas por plazos a lograr.

En tal sentido se recomienda constar con un documento explícito sobre los conceptos implicados, la justificación y fundamentación de la estrategia institucional para el empleo pedagógico de las TIC, tareas a largo, mediano y corto plazos encaminadas a la integración de las tecnologías a las distintas funciones sustantivas de la institución educativa, y no tan solo en el proceso de aprendizaje y enseñanza. 
2. Diseñar o rediseñar el modelo educativo de la institución, explicando los principios psicopedagógicos en que se fundamenta el empleo de las TIC y cómo éstas contribuyen en la mediación pedagógica profesor - estudiante, contenido de enseñanza - estudiante, estudiante - estudiante .

La búsqueda de un modelo educativo en que las TIC tienen una presencia significativa en varias direcciones entre ellas que optimice recursos, racionalice funciones, economice tiempo y satisfaga mejor las necesidades de aprendizaje y crecimiento de la generación net en formación.

3. Determinar el grado de aceptación, conocimiento, y habilidades de los miembros de la comunidad escolar, tanto los profesores, estudiantes como administrativos, tienen sobre la necesidad y posibilidad de empleo de las TIC en los procesos formales de educación y enseñanza. En otras palabras en qué medida los directivos, maestros, estudiantes y en general el personal de la institución conoce y hacen suyos en la práctica diaria las TIC para a partir de esto diseñar distintas estrategias y niveles de capacitación, desarrollo profesional y plantear las posibles metas a alcanzar en tal dirección.

4. Sensibilizar y capacitar de los maestros, y del personal implicado en las competencias profesionales que son necesarias para el trabajo en esta nueva modalidad en que los espacios tanto presenciales como a distancia (en línea) y sus posibles combinaciones exigen una actuación didáctica con el apoyo de recursos tecnológicos nunca antes considerados. Si importante resultan las competencias digitales, trascendentes son las competencias didácticas que exigen la integración de las TIC como parte del diseño educativo.

La capacitación debe ser precedida o acompañada de la sensibilización necesaria y suficiente para que "hagan suyo" un modelo educativo en que las TIC juegan un papel nunca antes considerado y que exige entre otras cosas una continua capacitación tanto teórica y mayormente práctica de acuerdo al modelo y su fundamentación psicopedagógica. Tanto en aspectos tecnológicos, al menos los necesarios, como pedagógicos, aquellos que tienen que ver con el diseño didáctico de los contenidos por los profesores en comunidades de aprendizaje.

5. Garantizar el desarrollo profesional continuo de los miembros de la comunidad educativa de modo tal que el empleo de las tecnologías de la información y de las comunicaciones (TIC) sea de acuerdo con el modelo y entre otros factores los objetivos educativos que se persiguen y en ningún momento como prácticas aisladas de profesores o grupos de profesores a iniciativa y criterios de ellos sin tener en consideración la visión y misión institucional, y la posibilidad de otras experiencias que de modo concomitantes se realizan en la institución y que pueden tener una misma población meta. El tal sentido la lógica del contenido de cada curso es un factor determinante a la hora de seleccionar el tipo de tecnología más apropiado para el desarrollo del mismo y el logro de los objetivos planteados. 
6. Seleccionar los nuevos maestros a partir de los criterios institucionales establecidos pero también por el nivel de desarrollo de competencias tecnológicas (digitales) y didácticas para el empleo de las TIC a los procesos educativos, o al menos de una actitud de aceptación que permita mediante los programas de desarrollo profesional de la institución ponerlo al día al respecto y exigirle por el cumplimiento de ello. Se pone en evidencia la necesidad de investigaciones sobre el nuevo perfil del profesor que sin duda alguna implica un conjunto de competencias tecnológicas y didácticas que hace algunos años no se tomaban en consideración.

7. Adoptar una plataforma electrónica probada y al nivel de las exigencias del desarrollo profesional de la comunidad educativa, bien comercial, de acceso libre o construido por la institución. La selección de una plataforma electrónica que satisfaga distintos criterios, desde los eminentemente económicos como aquellos que tienen que ver con las reales posibilidades de los estudiantes y de profesores para el desarrollo de los contenidos de los programas académicos, y el logro de los objetivos educativos planteados así como el desarrollo profesional de los maestros. Tal selección resulta clave para el buen desarrollo de los programas académicos institucionales en pos de la formación del ciudadano y profesional que la sociedad exige.

8. Incluir como elemento de apoyo o alternativos tantos recursos y aplicaciones tecnológicos como sean necesarios y respondan a la naturaleza lógica del contenido de la ciencia en cuestión como del código de comunicación propio del medio tecnológico. El empleo de una plataforma por buena que esta sea no debe excluir otros recursos que integrados a la misma permita la mejor presentación y desarrollo didáctico de los contenidos de enseñanza y interacción social, la cooperación y la comunicación entre estudiante y el profesor y los estudiantes entre sí para la construcción social de su conocimiento. El empleo de la plataforma seleccionada no debe excluir por supuesto el material escrito en una gama de presentaciones acorde con el nivel, tipo y perfil de la institución.

9. Desarrollar trabajo en equipo de profesores y especialistas en tecnologías para el diseño de los cursos ya sea estas "entregadas" a los alumnos mediante el internet a través de la página de la institución, alguna plataforma electrónica, un CD o bien DVD, o bien de entornos integrales de aprendizaje - enseñanza.

En tal sentido se sugiere la creación de un área o centro de apoyo institucional al trabajo didáctico del maestro en el diseño de los cursos para el logro de la integración de las TIC. El trabajo en equipo cooperativo de técnicos y docentes resulta clave para alcanzar los criterios de calidad tanto de tipo tecnológico como didácticos y de contenido.

10. Ofrecer servicio de orientación y soporte técnico tanto a estudiantes como los profesores y administrativos según horarios establecidos. Existencia de línea telefónica gratuita a disposición de los estudiantes. Tanto para consulta con el maestro como para solicitar ayuda tecnológica con el personal técnico se- 
gún horarios establecidos o bien en cuanto a la consulta tecnológica lo que se conoce por 7-24, los siete días de la semana, las veinticuatro horas del día.

11.Capacitar previamente los estudiantes antes de iniciar el curso o carrera. El desarrollo de un módulo cero o sesiones de orientación como se le conoce en algunas instituciones o bien sesiones de adiestramiento tecnológico y/o de auto estudio o estudio independiente para garantizar el correcto desempeño académico del estudiante así como orientación a la formación práctica en el área del conocimiento del curso. La lista de competencias digitales que exige los ambientes de aprendizajes que se diseñan en la institución resulta imprescindible para la capacitación de los nuevos grupos de estudiantes y también de profesores y administrativos.

12.Contar si las condiciones lo exigen dado el nivel de empleo de las TIC con un asistente del profesor con las competencias que exige el ejercicio de estos nuevos ambientes de aprendizajes en los que las TIC, ya de un tipo u otro son componentes esenciales para la mediación del profesor. La cifras varían y no se reconoce norma alguna al menos en la búsqueda realizada en instituciones latinoamericanas, no obstante alguno plantean la necesidad de que el maestro cuente con un asistente tecnológico cada 50 estudiantes.

13. Controlar la calidad del empleo de las TIC. Preocupación y ocupación por los indicadores de calidad de la aplicación de las TIC a la educación. Sugerencia de que se cuente con el equipo, oficina o centro de atención y control al proceso.

14.Estudiar mediante frecuentes encuestas el estado de opinión de los estudiantes, maestros y personal administrativo. Realización de encuestas y entrevistas que permiten monitorear el proceso y tomar medidas oportunas a tiempo. Confección, de portafolios de evidencias que permitan documentar y sistematizar el proceso y sus resultados.

\section{Conclusión}

A partir de los datos que nos sirven de punto de referencia y la reflexión lograda en las sesiones de trabajo (entrevistas, grupos focales, talleres de maestros, etc.) y tomando en consideración que:

- Los criterios recogidos cada uno en particular y sobre todo en su conjunto, reflejan el deber ser sobre el empleo de las TIC en la enseñanza y de la modalidad de educación a distancia según el grupo de expertos latinoamericanos participantes. Son criterios a nivel de institución, en otras palabras lo que la escuela, universidad o centro educativo debe tener en consideración para la incorporación y empleo adecuado de las TIC a la educación ya sea esta presencial o a distancia en tu totalidad o bien una combinación de ambas.

- Los criterios planteados manifiestan además el nivel de conocimientos y comprensión que tienen estas personas encuestadas sobre el empleo de las tecnolo- 
gías de aprendizaje, un reflejo de su nivel de desarrollo a partir del trabajo que realizan y los resultados que obtienen así como, de la experiencia acumulada y compartida en sus instituciones y con colegas del continente latinoamericano.

Las buenas prácticas en cuanto a la aplicación de las TIC tendrán éxito en la medida en que a nivel central, de institución, se tomen en consideración entre otros los criterios de calidad mencionados en el desarrollo de este trabajo. La adopción de medidas en cuanto a la integración de las TIC a nivel institucional es condición esencial para la correcta integración de éstas en los diferentes niveles de la escuela o universidad. En la medida en que a este nivel se adoptan y ponen en práctica determinadas decisiones se hace posible el desarrollo exitoso de la integración de las TIC a nivel micro de relación profesor - estudiante - contenido de enseñanza.

\section{Referencias bibliográficas}

ADELL, J. (1997). Tendencias en educación en la sociedad de las tecnologías de la información. EDUTEC. Revista Electrónica de Tecnología Educativa, 7. Recuperado en 1 de febrero de 2011 de http://www.uib.es/depart/gte/revelec 7.html

ALMANZOR, C. (2007). Importancia de la capacitación pedagógica del docente. Disponible en http://www.uag.mx/63/a04-02.htm

COLLINS-BROWN, E. (2001). Successful strategies for using a synchronous discussion in colleges courses[Estrategias exitosas para el uso de discusiones asincrónicas en cursos universitarios ]. Recuperado el 5 de octubre de 2011 de http://www.connectedcretivity.com/Treatise_EliCollinsBrown.doc

COORDINACIÓN DE EDUCACIÓN CONTINUA, ABIERTA Y A DISTANCIA. (2000). Desarrollo de ambientes de aprendizaje en educación a distancia. México.: Documento de Trabajo. Universidad de Guadalajara.

DFEE. (1999). Survey of Information and Comunications. Technology in Schools 1999. London: HMSO.

DFEE. (2000). Survey of Information and Comunications. Technology in Schools 2000. London: HMSO.

EDUCATION WEEK. (2004). Global Links: Lessons from the world. Technology Counts 2004. USA. American Educations's Newpaper or Record.

FERREIRO, R. (2011). NSU Informe de Investigación. North Miami Beach: Nova Southeastern University.

FERREIRO, R. (2006). Nuevas alternativas para aprender y enseñar. México: Trillas.

HERNÁNDEZ, S. (2008). El modelo constructivista con las nuevas tecnologías: aplicado en el proceso de aprendizaje [Versión electrónica]. En: Comunicación y construcción del conocimiento en el nuevo espacio tecnológico. Revista de la Universi- 
dad y Sociedad del Conocimiento (RUSC), 5(2), 26-35. Recuperado el 26 de febrero de 2011 de http://www.uoc.edu/rusc/5/2/dt/esp/hernandez.pdf

MENDOZA, J. (2003). e-learning, el futuro de la educación a distancia. Disponible enhttp://www.informaticamilenium.com.mx/Paginas/mn/ articulo78.htm

MÉXICO WORLD BANK LATIN.(2003). American and Caribbean Studies.Closing the gap in Education and technology. Washington.

NEWBERRY, B. (2001). Raising student social presence in online clases[El aumento de la presencia social de los estudiantes en los cursos en línea ]. World Conference on the WWW and Internet Proceedings. (No. de servicio de reproducción de documentos ERIC ED 466 611).

NEW YORK STATE EDUCATION DEPARTMENT (2007).Principles and operational criteria for good practice in distance higher education. Disponible en http://www.highered.nysed.gov/ocue/ded/practice.html

ORELLANA, A. (2009). Class size and interaction in online courses.En A. Orellana, T. L. Hudgins \& M. Simonson (Eds.), The perfect online course: Best practices for designing and teaching (pp. 117-135). Charlotte, NC, E.E.U.U.: Information Age Publishing.

ROGERS, E. M. (1995). Diffusion of innovations. N.Y: The Free Press.

SORENSEN, C. K. \& BAYLEN, M. D. (2009). Adapting the seven principles of good practice to web-based instructional environment.

\section{Correspondencia con el autor}

Ramón F. FERREIRO GRAVIE

Nova Southeastern University

1750 NE. $167^{\text {th }}$ Street. North Miami Beach

Florida 33162

Tel.: 1- 954-262-8680

E-mail: rferreir@nova.edu 